\title{
Whey protein isolate attenuates oxidative stress induced by intense exercise in trained cyclist men
}

\author{
W.T.S. Ton ${ }^{1}$, E.C. Santos ${ }^{2}$, J.C.B. Marins ${ }^{3}$ and R.C.G. Alfenas ${ }^{1}$ \\ ${ }^{1}$ Department of Nutritional Sciences, Universidade Federal de Viçosa, Brazil, ${ }^{2}$ Department of Animal Biology, \\ Universidade Federal de Viçosa, Brazil and ${ }^{3}$ Department of Sports Sciences, Universidade Federal de Viçosa, Brazil.
}

Muscular tissue is the major producer and the main target of reactive oxygen species (ROS) during exercise ${ }^{(1)}$. Excessive production of ROS impairs muscle recovery, leading to fatigue, compromising performance ${ }^{(2)}$. Whey and soy proteins may positively affect athletes' performance, due to their antioxidant activity ${ }^{(3,4)}$. The aim of this study was to compare the effect of isolate proteins on oxidative stress markers' levels in trained male cyclists.

This is a randomized crossover study, in which 10 trained male cyclists completed three 8-days-length experimental sessions. In each session the cyclists consumed a protein drink $(0.5 \mathrm{~g}$ protein isolate of whey or soy $/ \mathrm{kg}$ body weight $)$ or control. In the first and eighth days, the cyclists reported to the laboratory, $10 \mathrm{~h}$ fasted to exercise. The $45 \mathrm{~min}$ exercise protocol was performed in a cycloergometer applying loads of watts $/ \mathrm{kg}$ of body weight. Blood samples were collected at fasting state and after exercise to assess oxidative stress markers Figure 1: glutathione (GSH), total antioxidant capacity (TAC), glutathione peroxidase (GPx), superoxide dismutase activity (SOD), 8-isoprostane and thiobarbituric acid reactive substances (TBARS).
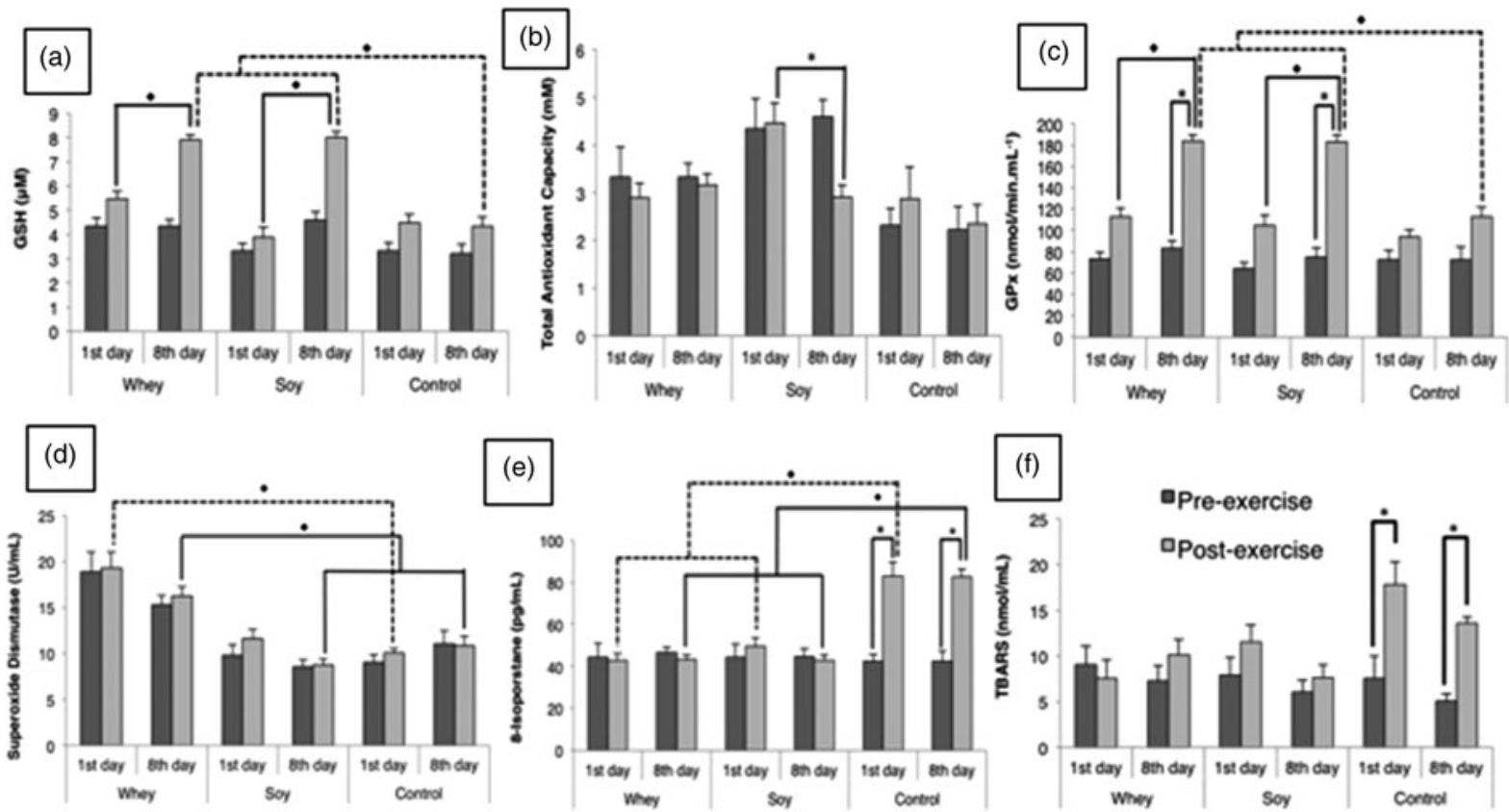

Fig. 1. a-f. Mean and SE biochemical parameters related to oxidative stress in the first and eighth days of consumption of whey protein isolate, soy protein isolate or control drinks, before and after exercising in a cycloergometer ( $\mathrm{n}=10$ cyclist men). Statistical significance $* \mathrm{p}<0.05$ by paired $t$ Student test. ${ }^{*} p<0.05$ by ANCOVA test, considering the baseline concentrations as covariants.

In conclusion, whey and soy proteins increased glutathione peroxidase activity and glutathione concentration and prevented postexercise lipid peroxidation. Whey protein increased superoxide dismutase activity compared with soy and control.

1. Peternelj TT \& Coombes JS (2011) Sports Med 41(12), 1043-1069.

2. Powers SK, Talbert EE, Adhihetty PJ (2011) J Physiol 589(Pt 9), 2129-2138.

3. Beermann C, Euler M, Herzberg J, Stahl B (2009) Eur Food Res Technol 229(4), 637-644.

4. Xu R, Liu N, Xu X, Kong B (2011) J Dairy Sci 94(8), 3739-3746. 